\title{
Determination of Intrinsic Mode and Linear Mode Coupling in Solar Microwave Bursts
}

\author{
Guangli Huang ${ }^{1,2}$, Qiwu Song ${ }^{1,2}$, and Jianping $\mathbf{L i}^{1,2}$
}

\begin{abstract}
An explicit equation of the propagational angle of microwave emission between the line-of-sight and the local magnetic field is newly derived based on the approximated formulae of nonthermal gyrosynchrotron emission (Dulk and Marsh 1982). The existence of the solution of propagational angle is clearly shown under a series of typical parameters in solar microwave observations. It could be used to determine the intrinsic mode and linear mode coupling in solar microwave bursts by three steps. 1) The mode coupling may happen only when the angle approximately equals to 90 degrees, i.e., when the emission propagates through the quasi-transverse region (Cohen 1960). 2) The inversion of polarization sense due to the weakly mode coupling takes place only when the transition frequency defined by Cohen (1960) is larger than the frequency of microwave emission, and an observable criterion of the weakly mode coupling in flaring loops was indicated by the same polarization sense in the two footpoints of a flaring loop (Melrose and and Robinson 1994). 3) Finally, the intrinsic mode of microwave emission is determined by the observed polarization and the calculated direction of local magnetic field according to the general plasma dispersion relation, together with the mode coupling process. However, a 180-degree ambiguity still exists in the direction of longitudinal magnetic field, to produce an uncertainty of the intrinsic mode. One example is selected to check the feasibility of the method in the 2001 September 25 event with a loop-like structure nearby the central meridian passage observed by Nobeyama Radio Heliograph and Polarimeters. The calculated angle in one footpoint (FP) varied around $90^{\circ}$ in two time intervals of the
\end{abstract}

Guangli Huang ${ }^{1,2}$, Qiwu Song ${ }^{1,2}$, and Jianping $\mathrm{Li}^{1,2}$

${ }^{1}$ Purple Mountain Observatory, Chinese Academy of Sciences (CAS), Nanjing, 210008, China

${ }^{2}$ Key Laboratory of Dark Matter and Space Astronomy, CAS, Nanjing, 210008, China maximum phase, which gives a direct evidence of the emission propagating through a quasi-transverse region where the linear mode coupling took place, while, the angle in another $\mathrm{FP}$ was always smaller than $90^{\circ}$ where the mode coupling did not happen. Moreover, the rightcircular sense at $17 \mathrm{GHz}$ was always observed in both two FPs during the event, which supports that the transition frequency should be larger than $17 \mathrm{GHz}$ in the first FP together with strong magnetic field of over 2000 Gauses in photosphere, where the weakly coupled case should happen. Moreover, there are two possibilities of the intrinsic mode in the two FPs due to the 180-degree ambiguity. 1) The emission of extraordinary (X) mode from the first FP turns to the ordinary $(\mathrm{O})$ mode in the two time intervals of the maximum phase, while, the X-mode is always emitted from the second FP. 2) The inversion from $\mathrm{O}$-mode to $\mathrm{X}$-mode takes place in the first FP, while the O-mode keeps in the second FP. If the magnetic polarities in photosphere and corona are coincident in this event, the intrinsic mode belongs to the second case.

Keywords flares; radio radiation; corona; magnetic fields; polarization; plasmas

\section{INTRODUCTION}

Radio polarization is generally considered as an important parameter for measuring the local magnetic field, as well as for determining the intrinsic mode of electromagnetic emissions from the local plasmas. The spatial resolvable data of Stokes I and V components are provided by a series of solar radio instruments in different frequencies, such as Nobeyama Radioheliograph (NoRH), Nancy Radio Heliograph (NRH), Siberian Solar Radio Telescope (SSRT), Owens Valley Solar Arrays (OVSA), and etc. Especially, high-quality images of 
Stokes I and V components at $17 \mathrm{GHz}$ are continuously recorded from 1992 up to date in $\mathrm{NoRH}$, and intensively studied by solar astronomers in all over the world. But only limited authors paid particular attention to the radio polarization observed by $\mathrm{NoRH}$.

In five events (Kundu et al. 2001), the NoRH images show direct evidence that the radio sources are compact bipolar loops: source sizes are less than 5", and three of the five events studied show closely spaced oppositely polarized components in the circular polarization maps. All five events are located directly over magnetic neutral lines in the photosphere, which is consistent with a single-loop scenario in which magnetic energy release and acceleration of nonthermal electrons are confined to a compact localized region. The polarization is analyzed in four microwave bursts observed by NoRH with one looptop (LT) and two footpoint (FP) sources ( $\mathrm{Su}$ and Huang 2004). The three sources in each given burst are always polarized in the same sense. This may be interpreted in terms of extraordinary mode (X-mode) emission, by taking into account the polarity of the underlying magnetic field and propagation effects, which may lead to inversion of the sense of polarization in the limbward FP and LT sources of the flaring loop. Huang and Lin (2006) found a quasi-periodic reversal between left-circular polarization (LCP) and right-circular polarization (RCP) in the LT source, five minutes before the 2002 April 21 flare observed by NoRH, and the polarization gradually turned to LCP. During this period, the polarization of the corresponding FP source maintained the RCP sense. It may be taken as evidence that magnetic energy is released or energetic particles are produced at the magnetic reconnection site in a quasi-periodic fashion.

Moreover, in the 2001 March 10 flare observed simultaneously by NoRH and SSRT, the emission at 17 $\mathrm{GHz}$ with LCP is generated in positive magnetic field polarity of the Michelson Doppler Imager (MDI) photospheric magnetogram. It is considered as an evidence of the ordinary mode (O-mode) emission and explained by strong anisotropy of the pitch angle distribution of the nonthermal electrons that responsible for the radio emission (Altyntzev et al. 2008). In a recent statistics of Huang et al. (2010), two footpoint emissions are compared in a total of $24 \mathrm{NoRH}$ events with loop-like structures. The polarization degrees in the two FPs are well correlated, while, they mostly have the same sense (RCP or LCP). There is a comparable proportion of the normal and abnormal events, which are defined as whether a stronger emission corresponds to a stronger or weaker polarization in the two FP sources (Kundu et al. 1995).

On the other hand, the observed polarization sense (RCP or LCP) may be reversed due to the linear mode coupling mechanism (Cohen 1960; Melrose and Robinson 1994; Zheleznyakov et al. 1996), on the basis of plasma dispersion relation under approximations of locally homogeneous corona and geometrical optics, which is widely used to analyze the polarization of solar radio emission (e.g., Peterova and Akhmedov 1974). When the emission propagating along the line-of-sight is nearly perpendicular to the local magnetic field (QT-region), the observed polarization sense is reversed in those frequencies below the transition frequency (weakly coupled), defined by Cohen (1960). Otherwise, the sense is unchanged in those frequencies above the transition frequency (strongly coupled). For two footpoint emissions with opposite magnetic polarities, they should show the same polarization sense in the weakly coupled case, but opposite polarization senses in the strongly coupled case (Melrose and Robinson 1994). However, it is not easy to judge if the observed emission does actually propagate through the QT-region, and the observations were not always compatible with that predicted by the theory (Mclean and Sheridan 1972; White et al. 1992).

It is predicted by Ramaty (1969) for the nonthermal gyrosynchrotron (GS) emission that the extraordinary mode (X-mode) is dominated in an opticallythin source, and the O-mode is only dominated in an optically-thick source. One case to observe the optically-thin O-mode emission in microwave band is caused by the thermal gyroresonance absorption at the third harmonic (Preka-Papadema and Alissandrakis 1988), the suppression of $\mathrm{X}$-mode emission, originating in the lower layers of the loop, will produce excess Omode radiation at the diskward foot of a flaring loop. This requires that the magnetic field is strong enough to bring the third harmonic layer inside the flaring loop. Thus, Alissandrakis et al. (1993) have found evidence for the O-mode emission in two classes of events. In one class the O-mode comes from the regions overlying the strong magnetic field, which can be interpreted in terms of the thermal gyroresonance absorption at the third harmonic. In the other class the entire burst emits in the O-mode, which may be attributed to high GS optical depth. The second case of optically-thin O-mode emission in microwave band was reported by Ledenev et al. (2002) in the 2000 July 14 event, due to the scattering of electromagnetic radiation during propagation through a plasma layer with developed Langmuir turbulence. The ordinary component is slightly lowered, while the extraordinary component undergoes the most effective scattering. The third case of optically-thin Omode emission in microwave band was suggested by White et al. (1992) and Gopalswamy et al. (1994), and the linear mode coupling may take place in the 
plasma current sheet with a transverse component of magnetic field. It was also reported by Vourlidas et al. (1997) that the O-mode emission in $4.7 \mathrm{GHz}$ observed by VLA in a solar active region, in contradiction with the gyroresonance models. The excess of the O-mode emission is attributed to the magnetic field configuration and the temperature inhomogeneities across the spot, and it may originate from the hotter penumbral loops.

Therefore, it is convenient to study the linear mode coupling and the intrinsic mode of electromagnetic emissions from the observed polarization sense and photospheric magnetic polarity. However, it is difficult to measure the local magnetic field of radio sources with lower plasma density in solar corona than that in the photosphere. For example, the radio emission at $17 \mathrm{GHz}$ is generated at least ten thousand kilometers above the photosphere, and the photospheric field lines may turn to different directions in solar corona. Section 2 shows one method to calculate the propagational angle of microwave emission between the line-of-sight and the local magnetic field, thus, to determine the linear mode coupling process and the intrinsic mode in solar microwave bursts. Section 3 analyzes one example observed by NoRH. Relevant discussions are given in Section 4.

\section{METHOD}

There are two equations derived in Huang (2006) for the coronal magnetic field strength $(B)$, the angle $(\theta)$ between the the coronal magnetic field and the lineof-sight (i.e., Eqs.(8) and (9) in Huang 2006), based on the nonthermal GS formulae in Dulk and Marsh (1982), with an error of about $30 \%$ in respect to the full expressions of nonthermal GS emission, for a limited range of electron spectral index, harmonic number, propagation angle etc. The detailed derivations and discussions are given in Huang (2006), and followed an earlier paper of Zhou and Karlický (1994).

$$
\begin{aligned}
& \log \left(\frac{\nu}{\nu_{B}}\right)=\frac{A_{1}+0.5 A_{2} \log \left(1-x^{2}\right)}{A_{3}}, \\
& \log \left(\frac{\nu}{\nu_{B}}\right)=\frac{A_{4}-0.071 x}{0.782-0.545 x}
\end{aligned}
$$

$$
A_{1}=-9.30+0.30 \delta+(1.30+0.98 \delta) \log \left(\frac{\nu}{\nu_{p}}\right)+\log T_{b \nu}
$$

$A_{2}=0.34+0.07 \delta$

$A_{3}=0.52+0.08 \delta$

$A_{4}=0.10+0.035 \delta-\log r_{c}$.

Here, $x=\cos \theta$, the electron gyrofrequency $\nu_{B}=$ $2.8 \times 10^{6} B$. All coefficients in Eq.(3)-(6) depend on five observable values, i.e., radio frequency $\nu$, the turnover frequency $\nu_{p}$, the brightness temperature $T_{b \nu}$ at a given frequency $\nu$, the polarization degree $r_{c}$, and the electron spectral index $\delta$, which is calculated by $\delta \approx$ $(1.22-\alpha) / 0.9$, here, $\alpha$ is the photon spectral index in the optically-thin part (Dulk and Marsh 1982). Thus, it is easy to cancel out the terms with $B$ in Eqs.(1) and (2), and to obtain a new equation of $x=\cos \theta$ as following.

$$
0.782 A_{1}-A_{3} A_{4}+\left(0.071 A_{3}-0.545 A_{1}\right) x+
$$

$$
+0.5 A_{2}(0.782-0.545 x) \log \left(1-x^{2}\right)=0 .
$$

The theoretical error of the approximations in Dulk and Marsh (1982) is better than 30\% in respect to the full expressions of nonthermal GS emission (Takakura and Scalise 1970), under the conditions of $2 \leq \delta \leq 7$, $\nu / \nu_{B} \geq 10$, the propagation angle $\theta>20^{\circ}$, the low cutoff energy $E_{0}=10 \mathrm{keV}$, and the emission is dominated by the extraordinary mode, but the accuracy worsens at $\delta>6$, especially, at extremes of $\theta$ and $\nu / \nu_{B}$ (Dulk $1985)$.

Now, we pay particular attention to the propagation angle $\theta$ in Eq.(7). When $\theta<90^{\circ}$, it means a positive magnetic polarity in respect to ordinary and extraordinary mode emission, together with LCP and RCP sense along the line-of-sight, respectively. In contrast, when $\theta>90^{\circ}$, it means that a negative magnetic polarity appears in the radio source, the emission with LCP sense along the line-of-sight will turn to the X-mode, and the emission with RCP sense along the line-of-sight will turn to the O-mode.

However, the polarization is always positive, and the angle $\theta$ is always smaller than 90 degrees in the approximated formulae of Dulk and Marsh (1982). Actually, the absolute degree of polarization must be the same at either side of $\theta=90^{\circ}$, but the sense of polarization is opposite in the two cases, and the polarization 
sense changes its sign around $\theta=90^{\circ}$ according to the general plasma dispersion relation. Hence, we cannot determine the exact direction of the longitudinal magnetic field with such a 180-degree ambiguity.

With typical values of $T_{b \nu}, r_{c}, \nu, \nu_{p}$, and $\alpha$ in microwave bursts, when $0 \leq x \leq 1$ or $0 \leq \theta \leq \pi / 2$, we find the left side of Eq.(7) varies monotonously with $x$ from positive values to negative values, which means that a unique solution possibly exists for Eq.(7). Figure 1 shows the solution of Eq.(7) with various values of $T_{b \nu}, r_{c}, \nu_{p}$, and $\alpha$, here, the radio frequency $\nu$ is fixed as $17 \mathrm{GHz}$ for NoRH. Thus, we can actually minimize the value of the left side of Eq.(7) to a small value (e.g., $10^{-2}$ ) to estimate the solution of $\theta$.

In principle, we may determine the intrinsic mode and linear mode coupling in solar microwave bursts by three steps.

1) At first, the mode coupling may happen only when the angle approximately equals to 90 degrees, i.e., when the emission propagates through the quasi-transverse region (Cohen 1960).

2) Secondly, the inversion of polarization sense due to the weakly mode coupling takes place only when the transition frequency defined by Cohen (1960) is larger than the frequency of microwave emission, and an observable criterion of the weakly mode coupling in flaring loops is indicated by the same polarization sense in the two footpoints of a flaring loop (Melrose and and Robinson 1994).

3) Finally, the intrinsic mode of microwave emission is determined by the observed polarization and the calculated direction of local magnetic field according to the general plasma dispersion relation, together with the mode coupling process. However, a 180-degree ambiguity still exists in the direction of longitudinal magnetic field, to produce an uncertainty of the intrinsic mode.

\section{ANALYSIS OF ONE NORH EVENT}

An M7.6 flare in active region NOAA 9628 (S21E01) start at 04:25:09 UT, peak at 04:34:00 UT, and end at 05:11:47 UT in 2001 September 25, is selected from the flare list in the web of NoRH. The full Sun observations of Nobeyema Radio Polarimeters (NoRP) at 1-35 GHz in the same event (Figure 2) are used to discuss the radiation mechanism of 17 and $34 \mathrm{GHz}$ observed by NoRH. It includes light curve, polarization, spectrum and turnover frequency during the event.

There are different polarization senses in different frequencies, such as the LCP sense in $1 \mathrm{GHz}$, and RCP sense at $17 \mathrm{GHz}$, which is consistent with the polarization at $17 \mathrm{GHz}$ observed by $\mathrm{NoRH}$ as shown in the left bottom panel of Figure 4. The spectra at three selected times show the typical features of nonthermal GS emission, but we still can't exclude the plasma emission that possibly existed at 1-2 GHz (i.e., the CD type with second peak in decimeter band by Nita et al. 2004). The turnover frequency increased fast in the rising phase, then, it varied in 10-15 GHz. Due to larger errors of peak frequency that fitted from only six frequencies of NoRP, we suppose that $35 \mathrm{GHz}$ always belongs to optically thin, but $17 \mathrm{GHz}$ is somehow close to the peak frequency in this event, which may cause an error in the calculated spectral indices of NoRH.

The left top panel of Figure 3 shows a bright soft Xray loop observed by Yohkoh/SXT in this event, overlaid by the contours of the $\mathrm{NoRH} / 17 \mathrm{GHz}$ brightness temperature (solid) and polarization degree (dashed). The soft X-ray emission in looptop (LT) is much stronger than almost symmetric emissions in two footpoints (FP1 and FP2), while, the microwave emissions in the two FPs are very asymmetric, with a large difference of almost one order of magnitude during the burst, as shown in the left bottom panel of Figure 3.

Therefore, we calculate the propagational angle $\theta$ from Eq.(7) in this event with the brightness temperature $\left(T_{b \nu}\right)$ at 17 and $34 \mathrm{GHz}$, and the polarization degree $\left(r_{c}\right)$ at $17 \mathrm{GHz}$ observed by NoRH, and the spectral index $(\alpha)$ calculated with the particular software of NoRH at 17 and $34 \mathrm{GHz}$ with different spatial resolutions, together with the peak frequency $\left(\nu_{p}\right)$ estimated by NoRP.

It is most interesting that the propagation angle $\theta$ in FP1 is much larger than that in FP2, and overlaid on the photospheric magnetogram of MDI in this event (see the right top panel of Figure 3). To confirm this result, we draw the time variation of the propagation angle $\theta$ in the two FPs in the right bottom panel of Figure 3. It is evident that $\theta$ reaches $90^{\circ}$ around the peak time in $\mathrm{FP} 1$ (the variation of $\theta>90^{\circ}$ is marked by dotted line), but $\theta$ in FP2 mostly varies around $20^{\circ}$. The calculated propagation angles in the two FPs just mean that there are opposite magnetic polarities in the two FPs during the impulsive phase of the event, i.e., negative in FP1 and positive in FP2.

It is predicted that the polarization degree in the optically thin limit is inversely proportional to the propagation angle from Figure 3d and Eq.(16) of Dulk and Marsh (1982), which seems to conflict with the results in Figure 4 that shows stronger polarization and larger propagation angle in FP1, while, weaker polarization and smaller propagation angle in FP2. The apparent discrepancy between the theoretical prediction and the results in Figure 4 may be caused by the asymmetric magnetic fields in the two FPs. The averaged photosphere magnetic field in FP1 is about 5-10 times larger 
than that in FP2, which may have stronger effect on the polarization degree than that of the propagation angle. The weak polarization commonly corresponds to the small magnetic field, and the calculations in Figure 3d of Dulk and Marsh (1982) should be obtained with the same magnetic field strength.

Moreover, the reason why the apparent photospheric magnetic polarities in the two FPs are opposite to the calculated coronal magnetic polarities may be not true due to the 180-degree ambiguity as mentioned above.

\section{DISCUSSIONS}

In the general plasma dispersion equation, the extraordinary mode (X-mode) emission along the line-of-sight corresponds to the observed right-circular polarization (RCP) emitted from a positive magnetic polarity, and the ordinary mode emission (O-mode) along the line-ofsight corresponds to the observed RCP emitted from a negative magnetic polarity. Note that the left-circular polarization (LCP) is detected as frequently as the RCP in solar radio observations (see the recent statistics of Huang and Nakajima 2009). In this case, the X-mode emission along the line-of-sight may correspond to the observed LCP emitted from a negative magnetic polarity, and the O-mode emission along the line-of-sight corresponds to the observed LCP emitted from a positive magnetic polarity (e.g., Altyntsev et al. 2008).

The key problem is that we may make mistake by simply using the photospheric magnetic polarities to determine the intrinsic mode of the electromagnetic waves detected by solar radio telescopes. If we use the observed photospheric magnetic polarities to determine the intrinsic modes in the 2001 September 25 flare, the result seems to be opposite to that using the calculated longitudinal magnetic polarities in this event. But, we still have a 180-degree ambiguity about the direction of longitudinal magnetic field in present case.

Regarding the linear mode coupling, the selected event is very closed to the central meridian passage (CMP), thus it is difficult to distinguish the diskward and limbward sources in the two FPs, and to judge the emission from which FP source propagate through the QT-region. We can't be simply neglect the mode coupling in the two FP sources nearby the CMP, which may be caused by a small deviation of the finite size of the FP sources from the CMP. Moreover, the observed polarization at $17 \mathrm{GHz}$ in this event has same RCP sense in both two FPs together with opposite magnetic polarities, which implies that the transition frequency should be larger than $17 \mathrm{GHz}$, i.e., in the weakly coupled case (Melrose and Robinson 1994).
The calculated angle between the local magnetic field and the line-of-sight in FP1 varied around $90^{\circ}$ at four times $\left(t_{1}, t_{2}, t_{3}\right.$ and $\left.t_{4}\right)$ of the impulsive phase (Figure 3), which gives a direct evidence of the FP1 emission propagating through a QT region where the linear mode coupling took place. While, the calculated angle in another FP was always much smaller than $90^{\circ}$, which implies that the FP2 emission does not propagate through the QT region. On the other hand, the observed photospheric magnetic field in FP1 is about one order of magnitude larger than that in FP2 (Figure 3 ), it is ready to compare the the transition frequency defined by Cohen (1960) and the observed frequency $(17 \mathrm{GHz})$ of NoRH in both two FP sources, and it is confirmed that the mode coupling in FP1 actually belongs to the weakly coupled case. Moreover, the calculated angle between the line-of-sight and the local magnetic field becomes large with increasing of radio emissions, which just means an enhancement of the transverse magnetic field during the burst, and it may support the existence of the QT-region.

Now, we try to understand the intrinsic mode of the two FP emissions in this event. From the NoRP data as mentioned above, the $35 \mathrm{GHz}$ emission is located in the optically-thin region, which belongs to the $\mathrm{X}$ mode according to Ramaty's theory (1969), while, the $17 \mathrm{GHz}$ emission is close to the peak frequency and it may belong to $\mathrm{X}$-mode or $\mathrm{O}$-mode. If the angle $\theta<90^{\circ}$ or the longitudinal magnetic field is positive, when the $17 \mathrm{GHz}$ emission in FP1 propagates through the QTregion, where the intrinsic mode with the $\mathrm{RCP}$ sense should be reversed from the $\mathrm{X}$-mode to the O-mode in the two time intervals of the impulsive phase. The reversion from the $\mathrm{X}$-mode to the $\mathrm{O}$-mode took place at $t_{1}$ and $t_{3}$, and the reversion from the $\mathrm{O}$-mode to the $\mathrm{X}$-mode took place at $t_{2}$ and $t_{4}$. While, the $17 \mathrm{GHz}$ emission in FP2 does not propagate through the QT region, and the intrinsic mode in FP2 always belongs to the X-mode with the RCP sense. On the other hand, due to the 180-degree ambiguity, the angle $\theta$ may equal to $180^{\circ}$ minus the calculated value. Thus, the intrinsic mode in FP1 is just reversed from the O-mode to the Xmode at $t_{1}$ and $t_{3}$, and the reversion from the $\mathrm{X}$-mode to the O-mode took place at $t_{2}$ and $t_{4}$. While, the 17 $\mathrm{GHz}$ emission in FP2 always belongs to the O-mode.

Finally, there are two results with something new in this paper to be summarized as follows.

1) According to the theoretical prediction of Melrose and Robinson (1994), for two FP emissions with opposite magnetic polarities (such as the event in this paper), they should show the same polarization sense in the weakly coupled case, but opposite polarization senses in the strongly coupled case. The problem is that 
the flare is at the central meridian, and there should be rough symmetry in the ray paths to Earth. Hence, the first new result in this paper is just to give an evidence of the weakly mode coupling near by the central meridian, which is consistent with the theoretical prediction of Melrose and Robinson (1994).

2) Secondly, we have derived a new equation of $\cos \theta$ based on the earlier paper of Huang (2006), and shown the existence of the solution of this equation under some typical parameters of microwave bursts, which should be helpful for studying the model coupling and coronal magnetic field quantitatively. Though the uncertainty of the $\operatorname{sign}$ of $\cos \theta$ does exist, but it does not affect the prediction of the mode coupling around $\theta=90^{\circ}$.

Regarding the problem of intrinsic modes, we may compare the extrapolation of photosphere magnetic field with the calculated coronal magnetic field, and determine the exact direction of coronal magnetic field, which we plan to do in a forthcoming paper. In this paper, if we believe that the magnetic polarities in photosphere and corona are commonly coincident, the intrinsic modes can be determined therein.

The remained problem that the authors could not solve so far is that the uncertainty of peak frequencies in different locations. It is expected to study this problem when the new instruments (such as FASR and Chinese radio heliograph with multiple frequencies) are completed in near future.

The study is supported by two NFSC projects respectively with No.11073058 and 10833007, as well as the "973" program with No.2011CB811402. The authors would like to thank the NoRH teams for their dedicated data preparing and preprocessing.
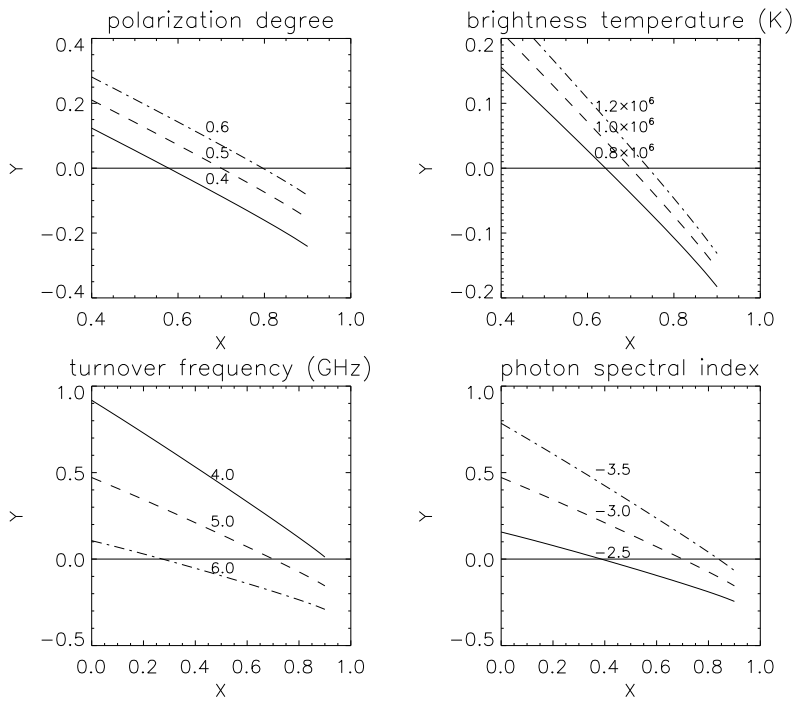

Fig. 1 Left top panel: the solution of Eq.(7) with different polarization degrees of 0.4 (solid), 0.5 (dashed), and 0.6 (dot-dashed). Left bottom panel: the solution of Eq.(7) with different brightness temperatures of $0.8 \times 10^{6} \mathrm{~K}$ (solid), $1.0 \times 10^{6} K$ (dashed), and $1.2 \times 10^{6} K$ (dot-dashed). Right top panel: the solution of Eq.(7) with different turnover frequencies of 4.0 (solid), 5.0 (dashed), and $6.0 \mathrm{GHz}$ (dot-dashed). Right bottom panel: the solution of Eq.(7) with different photon spectral indices of -2.5 (solid), -3.0 (dashed), and -3.5 (dot-dashed) The other parameters are always fixed as the middle value in their varied ranges for all the panels. 

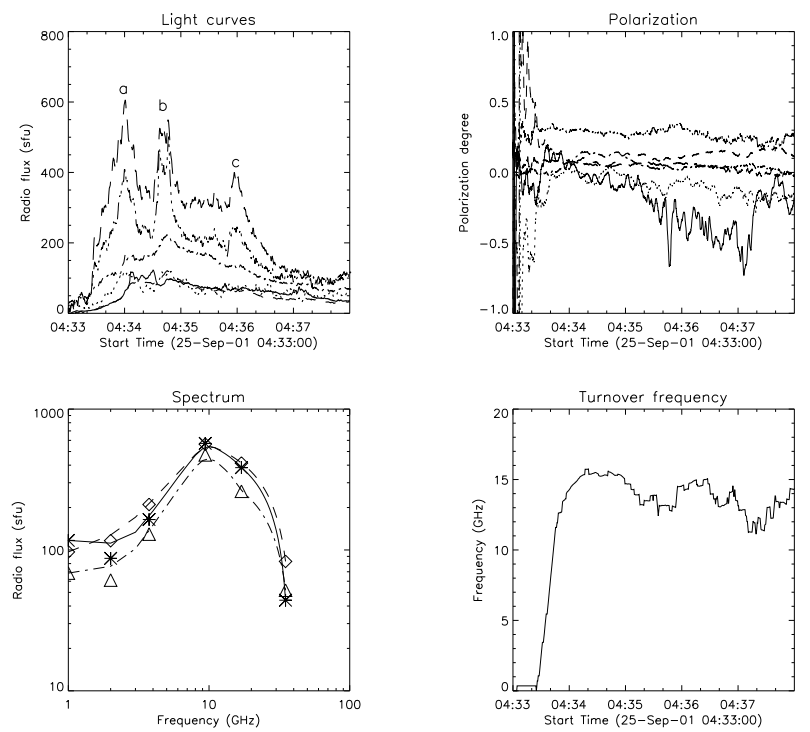

Fig. 2 Left top panel: Light curves of $1 \mathrm{GHz}$ (solid), $2 \mathrm{GHz}$ (dashed), 3.75 GHz (dot-dashed), $9.375 \mathrm{GHz}$ (long dashed), $17 \mathrm{GHz}$ (dot-dot-dashed), and $35 \mathrm{GHz}$ (dotted). Right top panel: Polarization degrees of $1 \mathrm{GHz}$ (solid), $2 \mathrm{GHz}$ (dashed), 3.75 GHz (dot-dashed), $9.375 \mathrm{GHz}$ (long dashed), $17 \mathrm{GHz}$ (dot-dot-dashed), and $35 \mathrm{GHz}$ (dotted). Left bottom panel: Spectra at three selected times as marked by 'a' (solid), 'b' (dashed), and 'c' (dot-dashed) in the left top panel. Right bottom panel: Time evolution of the turnover frequency.
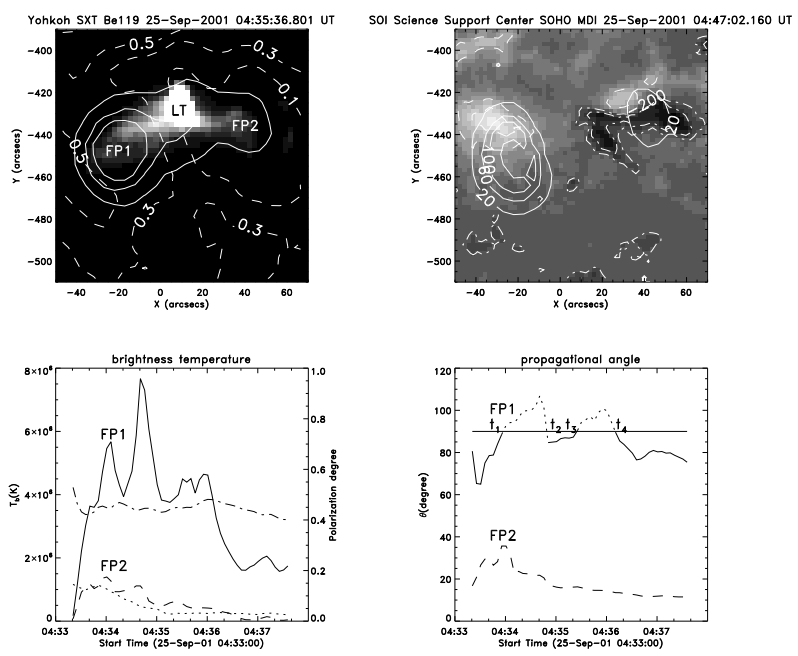

Fig. 3 Left top panel: Yohkoh/SXT image overlaid by NoRH/17GHz contours of $0.1,0.3$ and 0.5 maximum brightness temperature (solid) and polarization of $0.1,0.3$, and 0.5 (dashed). Left right panel: MDI magnetogram overlaid by the calculated propagational angle of 20,60, and 100 degree (solid), and magnetic strength of 1500 and 2500 Gauss (dashed), -200 and -800 Gauss (dot-dashed). Left bottom panel: the light curve and polarization degree of $\mathrm{NoRH} / 17 \mathrm{GHz}$ in FP1 (solid and dot-dashed, respectively) and FP2 (dashed and dotted, respectively), the position of FP1 and FP2 is marked in the left top panel. Right bottom panel: the time evolution of the propagational angle in FP1 (solid and dotted) and FP2 (dashed), the horizontal line marks the angle of 90 degrees. 


\section{References}

Alissandrakis, C. E., Nindos, A., and Kundu, M. R.: 1993, Solar Phys. 147, 343

Altyntsev, A. T., Fleishman, G. D., Huang, G. L., and Melnikov, V. F.: 2008, Astrophs. J. 677, 1367

Cohen, M. H.: 1960, Astrophs. J. 131, 664

Dulk, G. A. and Marsh, K. A.: 1982, Astrophs. J. 259, 350

Dulk, G. A.: 1985, ARA\&A 23, 169

opalswamy, N., Zheleznyakov, V. V., White, S. M., and Kundu, M. R.: 1994, Solar Phys. 155, 339

Huang, G. L.: 2006, Solar Phys. 237, 173

Huang, G. L. and Lin, J.: 2006, Astrophys. J. 639, L99

Huang, G. L. and Nakajima, H.: 2009, Astrophys. J. 696, 136

Huang, G. L. and Nakajima, H.: 2009, Astrophys. J. 702, 19

Huang, G. L., Song, Q. W., and Nakajima, H.: 2010, Astrophys. J. 723, 1806

Kundu, M. R., White, S. M., Shibasaki, K., Sakurai, T., and Grechnev, V. V.: 2001, Astrophys. J. 547, 1090

Kundu, M. R., Nitta, N., Shibasaki, K., Enome, S., Sakao, T., Kosugi, T., and Sakurai, T.: 1995, Astrophys. J. 454, 522

edenev, V. G., Tirsky, V. V., Tomozov, V. M., and Zlobec, P.: 2002, Astronom. Astrophys. 392, 1089

Mclean, D. J., and Sheridan, K. V.: 1972, Solar Phys. 26, 176

Melrose, D. B, and Robinson, P. A.: 1994 Astron. Soc. of Australia, Proc. 11, 16

Nita, G. M., Gary, D. E., and Jeongwoo Lee: 2004, Astrophys. J. 605, 528

Peterova, N. G., and Akhmedov, Sh. B.: 1974, Soviet Astron. 17, 768

Preka-Papadema, P., and Alissandrakis, C. E.: 1988, Astronom. Astrophys. 191, 365

Ramaty, R.: 1969, Astrophys. J. 158, 753

Su, Y. N. and Huang, G. L.: 2004, Solar Phys. 219, 159

Takakura, T., and Scalise, E.: 1970, Solar Phys. 11, 434

ourlidas, A., Bastian, T. S., and Aschwanden, M. J.: 1997, Astrophys. J. 489, 403

hite, S. M., Thejappa, G., and Kundu, M. R.: 1992, Solar Phys. 138, 163

Zheleznyakov, V. V., Kocharovsky, V. V., and Kocharovsky, Vl. V.: 1996, Astron. Astrophys. 308, 685

Zhou, A. H., and Karlický, M.: 1994, Solar Phys. 153, 441

This manuscript was prepared with the AAS LATEX macros v5.2. 